Historic, Archive Document

Do not assume content reflects current scientific knowledge, policies, or practices. 



$$
\begin{gathered}
\text { PRICE LIST } \\
\text { No. } 15
\end{gathered}
$$

\title{
HUTCHINSON NURSERIES
}

\section{Kearney, Nebraska}

\author{
ESTABLISHED 1911.
}

GROWERS OF CHOICE FRUITS AND

ORNAMENTALS FOR WESTERN

\author{
PLANTERS
}

MINMBERS

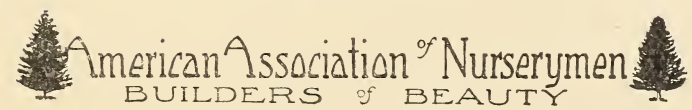



We thank you for your very liberal patronage in the past and assure you we shall do everything we possibly can to please you in the future. We have beautiful, healthy trees and shrubs that are sure to please. Our grades are the highest and all orders are packed carefully by experienced men. We guarantee every order to reach you in prime planting condition. Satisfaction or your money back is our broad guarantee if you will return any stock that does not suit you at once.

State Entomologists Certificate of Inspection.

Our growing stock is inspected each year and a certificate of inspection goes with each shipment showing that stock is free from disease.

\section{Terms of Payment.}

Those ordering direct from catalogue should send full remittance with order. Your personal check is good.

\section{Prepayment.}

We prepay all mailable size orders of $\$ 3.00$ or over. Less than this amount, we require $25 \%$ adiditional to cover postage and packing. Ail freight or express orders are prepaid if for $\$ 10.00$ or over. Where orders are sent C. O. D., we require that customers pay the small sum required for collecting and return of money.

\section{Replacing Stock that Fails to Grow.}

Most folks are reasonable about this. We will not let you be dissatisfied with our adjustments. We are always glad to replace anything free that does not make a start if it has had good care. If the season is unfavorable and the stock started to grow but dry hot weather kilied it or some other cause that the nurseryman could not help, we think a half-price adjustment is about right.

\section{Shipping Season.}

Spring shipments begin to move about March 25th and continue until about May 25th. Fal! shipments usually start with the perennials, about the 1st of September and continue until ground frcezes, about the 1 st of December. 


\section{QUANTITY RATES.}

1,000 rate.

6 at dozen rate. 50 at 100 rate. 500 at

\section{NOTICE.}

The varieties listed in this price list are especially suitable for Nebraska. Northern Kansas, Southern South Dakota and Colorado. We shall be glad to furnish other varieties as listed in our catalogue but we especially recommend the varieties as given in this price list which are the cream of the new varieties and the old standbys.

\section{Apples and Crabs}

Our apples are vigorous, healthy trees with splendid root system. Sure to please you.

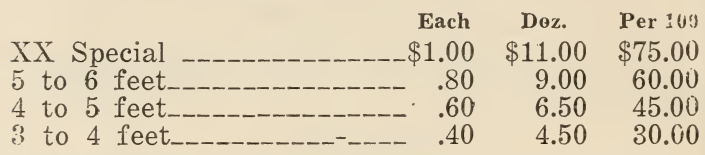

Winter Varieties: Delicious, Jonathan, Ben Davis, Black Ben Davis, Stayman Winesap, Missouri Pippin, Winesap, North West Greening and Janet. Fall Varieties: Wealthy, Maiden Blush. Surnmer: Early Harvest, Yellow Transparent, Duchess of Oldenburg. Crabs: Whitney, Florence, Hyslop, Red Siberian.

\section{Cherries and Comnass Cherry.}

Our cherries are budded on Mahaleb and will not sprout from roots. A standard Nebraska fruit.

\section{Each Doz. Per 100}

XX Special _-_______ $\$ 1.50 \quad \$ 16.00 \$ 120.00$ 4 to 6 feet, No. 1 grade____ $1.25 \quad 14.00 \quad 95.00$ 3 to 5 feet, nice trees _____ $1.00 \quad 11.00 \quad 75.00$

Varieties. Early Richmond. Large Montmorency, Wragg. Compass Cherry-Plum.

\section{Plums.}

Each Doz. Per 100

XX Special _-_-_-_-_-_\$1.50 \$16.50 $\$ 125.00$

5 to $T$ feet, No. 1

4 to 5 feet, nice trees____ $1.00 \quad 11.00 \quad 80.00$

3 to 4 feet, good trees_._. $\quad .75 \quad 9.00 \quad 60.00$ 
Varieties: Wyant, Wolf, Lombard, Abundance, Omaha. Hansen Hybrid Varieties: Waneta, Hanska and Sapa.

\section{Peaches.}

Recor.mended for trial only in this section. 4 to 6 feet, good trees_-_- $75 \%$ each. $\$ 8.00$ per doz. Hale.

Varieties: Elberta, Crawford's Early, J. H.

\section{Pears.}

Each Doz. Perivo XX Special _--_--_-_-_-_--_\$1.75 $\$ 20.00 \$ 150.00$ 5 to 6 feet___._- $1.50 \quad 16.00 \quad 120.00$ 4 to 5 feet_-_-_-_-_-_-_- $1.25 \quad 14.00 \quad 95.00$

Varieties: Flemish Beauty, Clapp's Favorite, Kieffer. Dwarf Duchess: 4 to 5 feet, $\$ 1.50$ each; \&16.00 per dozen.

\section{Russian Mulberry.}

5 to 6 feet

Each

Dozer

4 to 5 feet $75 c$ $\$ 8.00$ $50 c$ 5.00

Russian Apricots.

5 to 6 feet, select

Each Dozen $\$ 1.25 \$ 14.00$

Grapes, Concord.

No. $1-2$

Dozen

Hundred $\$ 3.00$ $\$ 18.00$ No. $1-1$ year

2.00

12.00

Grapes, Other Varieties.

Dozen

Hundred $\$ 4.00$

No. $1-2$ year 3.00 $\$ 30.00$ 22.50

Varieties: Niagara (white), Worden (black). Grapes, Special

Ecta, black and Lucile, red, extra hardy. Each

Dozen No. 1 50 c $\$ 5.50$

\section{Currants.}

Each Doz. Per 100 No. 1 -__ $\$ 4.00 \quad \$ 30.00$ Holland.

Varieties: Cherry, Fay's Prolific, Long Bunch 
Gooseberries.

Each Doz. Per 10?

No. 1 Varieties: Downing, Oregon Champion. $\$ 30.00$

Houghton Gooseberries $35 \phi \quad \$ 3.00 \quad \$ 20.00$

Raspberries, Blackberries, Dewberries.

Raspberries: Black, Cumberland and Kansas. Red, Columbian.

Blackberries: Snyder and Mersereau.

Dewberries: Lucretia.

No. 1

Two year Raspberries $-15 \& \quad \$ 1.50$

\section{Strawberries, June Bearing.}

In bunches of 25 plants.

Per 25 Per 100 Per $10 \jmath 0$ Senator Dunlap and Gibson_-_50 $\$ \quad \$ 1.50 \quad \$ 12.00$ Everbearing Strawberries.

Pe. 25 Per 100 Per 1000

Champion, Progressive

and Superb-__-_-_ $\$ 1.00 \quad \$ 3.00 \quad \$ 25.00$

\section{Miscellaneous.}

Asparagus, No. 1

Dozen

Per 100 $\$ .50$

Juneberry 5.00

Horse Radish _-____-__-__-__-_ 1.25

Rhubarb, the finest

1.50

$\$ 2.50$

\section{Shade and Ornamental Trees.}

Note: In lots of 10 to 50 of same variety and grade, discount prices $10 \%$. In lots over bo you may discount $15 \%$.

$\begin{array}{rrrrr}4-5 & 5-6 & 6-8 & 8-10 & 10-12 \\ \text { feet } & \text { feet } & \text { feet } & \text { feet } & \text { fret }\end{array}$

Ash, Am. White

Box Elder $\$ .75 \$ 1.00 \$ 1.75 \$ 3.00$

Catalpa Speciosa

$\begin{array}{llll}.50 & .75 & 1.75 & 3.00\end{array}$

Elm, Am. White_____\$.75

Elm, Moline

$\begin{array}{lll}.75 & 1.25 & 1.75\end{array}$

$\begin{array}{llll}1.00 & 1.50 & 2.00 & 3.50\end{array}$

Elm, Vase Shaped.

Catalpa Bungeii

2.00

Hackberry $-2.00$

Linden, American 1.00

Locust. Thornless

$\begin{array}{lll}3.00 & 3.00 & 4.00 \\ 1.50 & 2.00 & 3.00 \\ 1.50 & 2.00 & 3.00 \\ 1.00 & 1.50 & 2.00\end{array}$


Maple, Hard or Norway

Maple, Wiers Cut Leaf

Maple, Soft or Silver-

Mountain Ash

Oak, Pin -

Olive, Russian -

Poplar, Boleana _-_-- . .75

Poplar, Volga --_----

Poplar, Norway -----

Poplar, Silver Leaf_--

Sycamore

Willow, Niobe

Walnut, Black

$\begin{array}{rrrr}1.00 & 2.25 & & \\ .50 & 1.00 & 2.50 & \\ 2.00 & 3.00 & & \\ 3.00 & 4.00 & & \\ 1.50 & 2.00 & & \\ 1.00 & 1.75 & 3.00 & \\ 1.00 & 1.25 & 1.50 & \\ .40 & .75 & 1.00 & 2.00 \\ 1.00 & 1.50 & 2.00 & \\ 1.00 & 1.50 & 2.50 & \\ 1.00 & 1.50 & 2.50 & \\ 1.50 & & & \end{array}$

Forest Tree Seedlings.

Ash, Box Elder, Russian Mulberrv. Catalna Speciosa, Black Locust, Honey Locust, Elm. Sold only in lots of 100 of each variety.

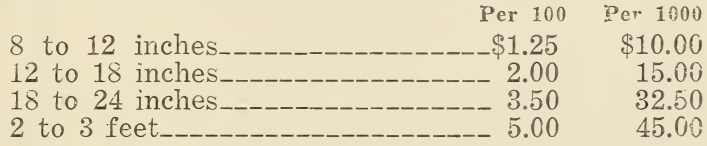

Russian Olive.

Per 100 Per 1000

8 to 12 inches

12 to 18 inches

18 to 24 inches_-_-_-_-_-_-_-_ 10.00

2 to 3 feet__________________-_1200

Norway Poplar (Sudden Saw Log) 1 Year.

Each Per 10 Per 100

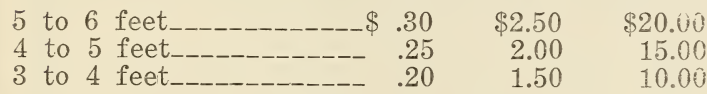

Hedging.

$\begin{array}{lrrrr} & 8-12 & 12-18 & 18-24 & 2^{2-3^{3}} \\ \text { Barberry, Japanese --- } & \$ 30.00 & \$ 40.00 & \$ 55.00 \\ \text { Buckthorn --------- } & 15.00 & 20.00 & 25.00 \\ \text { Lilac, Purple----- } & 40.00 & 50.00 & 60.00 \\ \text { Privet A.R.No. Hardy } \$ 10.00 & 15.00 & 20.00 & 25.00 \\ \text { Honeysuckle, Bush ---- } & 20.00 & 30.00 & 40.00 \\ \text { Spirea, Van Houtte--- } 12.00 & 25.00 & 35.00 & 50.00 \\ \text { Odessa Tamarix ----- } & & . & 40.00\end{array}$

Spirea Anthony Waterer and

Spirea Froebellii_-_-- $40.00 \quad 55.00$ 


\section{The Proper Distance Apart for Planting Hedge.}

Barberry 8 to 12 inches. Privet 6 to 16 inches. Spirea Van Houtte and Honeysuckle 1 to $2^{1 / 2}$ feet. Lilac 2 to 3 feet. Buckthorn 10 to 12 inches. Tamarix 2 feet. Spirea A. W. and? Spirea Froebellii, 8 to 12 inches.

Plants to form a low hedge should be planted more closely than when it is desired to have a high hedge or screen.

\section{Ornamental Shrubs.}

Altheá, 2-3 ft. $\$ .75,3-4 \mathrm{ft.}$

Almond, $18-24$ in. $\$ 1.00,2-3 \mathrm{ft}$.______-_. 1.20

Barberry Thunbergii, $18-24$ in. $\$ .75,2-3$ ft.-- 1.00

Buckthorn, 2-3 ft. \$.75, 3-4 ft._-______-_ 1.00

Buffalo Berry, 2-3 ft.-_-__-___-_._- . 75

Calycanthus, Sweet Scented Shrub, 2-3 ft.--- 1.00

Caragana, 2-3 ft. \$.75, 3-4 ft._-__-_-_-_-_ 1.00

Buddelia, Butterfly Bush, No. 1__-_-_-_-_- 1.00

Cotoneaster, 18-24 in. $\$ .75,2-3$ ft._-__-_-_- 1.00

Currant Flowering, 2-3 ft. \$.75, 3-4 ft.-_-_-. 1.00

Deutzia, $18-24$ in. \$.75, 2-3 ft.

Desmodium, Pea Shrub 18-24 in. \$.75, 2-3 ft. 1.00

Dogwood, 2-3 ft. $\$ .75,3-4 \mathrm{ft}$. - $\$$

Elder, $2-3 \mathrm{ft}$. $\$ .75,3-4$ ft._-_-_-_-_-- 1.00

Forsythia, 2-3 ft. $\$ .75,3-4$ ft.--

Smoke Tree, Fringe, $3-4$ ft. $\$ 1.00,4-5 \mathrm{ft}$.--- 1.25

Honeysuckle, Bush, $2-3 \mathrm{ft}$. $\$ .75,3-4 \mathrm{ft}$.

Flowering Quince, No. 1__- 1.00

High Bush Cranberry, 2-3 ft. $\$ 1.00,3-4$ ft.-- 1.25

Hydrangea, P. G. Tree Form, 3-4 ft._._- 1.50

Hycirangea, June Blooming, 2-3 ft..-_-_--- 1.25

Hydrangea, P. G., 2-3 ft. $\$ 1.00,3-4 \mathrm{ft}$.

Lilacs, purple and white, $2-3 \mathrm{ft}$. $\$ .75,3-4 \mathrm{ft} .--100$

Lilacs, Persian, $2-3 \mathrm{ft}$. $\$ 1.00,3-4 \mathrm{ft}$.--_-_--- 1.25

Jilacs, Named, $2-3 \mathrm{ft}$. $\$ 1.50,3-4 \mathrm{ft}$.---.--- 2.00

Privet, Specimen, 2-3 ft. $\$ .75,3-4 \mathrm{ft}$.

Mock Orange, Philadelphus, $2-3 \mathrm{ft} \$ .75,3-4 \mathrm{ft} .1 .00$

Mock Orang'e, Dwarf, $2-3$ ft. $\$ 1.00,3-4 \mathrm{ft}$.-- 1.25

Snowball, $2-3$ ft. $\$ 1.25 .3-4 \mathrm{ft}$.

Snowberry, red and white, $2-3 \mathrm{ft} . \$ .75,3-4 \mathrm{ft} .1 .00$

Spirea Arguta, $2-3 \mathrm{ft}$. $\$ 1.00,3-4 \mathrm{ft}$.

Spirea Anthony Waterer, $12-18$ in. $\$ .75,18-241.00$

Spirea Froebellii, $12-18$ in. $\$ .75,18-24$ in.--- 1.00

Spirea Thunbergii, $12-18$ in. $\$ 1.00,24-30$ in_- 1.25

Sprea Van Houtte, $2-3$ ft. $\$ .75 .3-4$ ft.----- 1.00 
Spirea Sorbifclia. $2-3 \mathrm{ft} . \$ 1.00,3-4 \mathrm{ft}$.

Spirea Billardii, 2-3 ft. $\$ .75,3-4 \mathrm{ft}$. --_-_- 1.00

Spirea Bethlemensis, $18-24$ in. $\$ .75,2-3 \frac{1}{1 / 2} \mathrm{ft} .1 .00$

Spirea, Ninebark, $2-3 \mathrm{ft}$ \$.75, 3-1 ft.-_-_-_- 1.00

Sumac, 2-3 ft. $\$ .75,3-4 \mathrm{ft.}$._-___-_-_-_-_ 1.00

Tamarix, 3-4 ft. $\$ .75,4-5 \mathrm{ft}$.

Viburnum Sterillis, $2-3$ ft. $\$ 1.00,3-4 \mathrm{ft} . \ldots+\ldots 1.25$

Weigelia, 2-3 ft. $\$ 1.00,3-4$ ft._-___-_-_-_ 1.25

Note: Where extra heavy shrubs are required for Landscape work, we can furnish at trom $\$ 1.50$ to $\$ 2.00$ each according to age and value of specimen.

\section{Shrubs-Tree Form.}

3 to 5 feet, each

Flowering Crab, Tree Lilac, Flowering Plum, Purple Leaf Plum, Red Bud or Judas Tree and Flow ering Thorn.

\section{Bedding Roses.}

Hybrid Perpetual: American Bøauty (r); General Jacqueminot (r); Frau Kari Druschki (w); Marshall P. Wilder (r); Mrs. John Laing: (p); Ulrich Brunner (r); Soliel d'Or, reddish gold.

Everblocming Hybrid Tea: Gruss an Tepletz (r), K. A. Victoria (w), Killarney (p), La France. (p), Los Angeles, flame pink, shaded gold each \$1.50; Mrs. Aaron Ward (y), Caroline Testout (p), Ophelia, salmon flesh color, shaded rose, Radiance, carmine rose, Ped Radiance Sunburst $(y)$.

June Blooming and other roses: Persian Yelow, Rosa Rugosa. Hugonis, \$2.50 each, (earliest yellow). Madam Plantier, inest June white and excellent or cemetery planting. noted:

Prices of all above roses unless otherwise $\begin{array}{lrr}\text { Sirong } & \text { Each } & \text { Doz. } \\ \text { Good field grown plants_-_-_-_-_- } \$ 1.25 & \$ 1.00 & 10.00\end{array}$

\section{Climbing Roses.}

Crimson Rambler, Paul's Scarlet Climber. White Dorothy Perkins, Dorothy Perkins, Yellow 
Rambler, Cl. Americun Beauty, blooms all summer, Dr. Van Fleet. $\$ 1.25$.

Prices of above unless otherwise noted, $\$ 1.0 \mathrm{C}$ each., $\$ 10.00$ per dozen.

\section{Vines.}

Clematis Jackmanii Furple Clematis Henryii, Large White_-___-__-_-- 1.50 Clematis Paniculata, Smali Sweet Scented_- $10 \%$ Honeysuckle, all varieties

Anıpelopsis. Quinquefolia, Woodbine .75 Ampelopsis, Engelmannii, hardy climbs smooth walls .75 Ampelopsis, Veitchii, (Boston Ivy) semi-hardy 1.00 Wisteria, Purple and White Bignonia, Trumpet Creeper. 1.0() Climbing' Bitter'sweet

\section{Perennial Plants.}

Doz. Each Ht. In.

Asters, Daisv-like Flower___ $\$ 4.00$ Bajy"s Brealin 4.00

Bleeding Heart 10.00

Canterbury Bells 4.50

Achillea, The Pearl

4.00

Aquilegia (Columbine) 4.00 Coreopsis

Daisies, Shasta 4.00 4.00

Chrysanthemums, (Spring delivery

$$
\text { only) }
$$

De

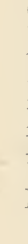

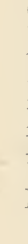

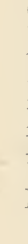

Gaillardia

Tiper Lily

Lily of the Valley

Hardy Pinks

Mallow Marvels

Hollyhocks

Poppy

\section{isenton (Japanese}

Bell Flower')

phinium, (Larkspur)

$\$ .35 \quad 24-36$

.3524

$1.00 \quad 18$

$.40 \quad 18-24$

$.35 \quad 24$

$.35 \quad 24$

$.35 \quad 24$

$.35 \quad 24$

$.40 \quad 30$

$.35 \quad 24-35$

$.40 \quad$, .18

.3518 


\section{Peonies.}

The Queen of the Garden. They last for a full month if early and late varieties are selected.

\section{Early White:}

Festiva Maxima

Mme. De Vernville

Midseason White:

Duchess de Nemours_-_-_-_-_-_-_-_-_-- 1.00

\section{Late White:}

Couronne de Or._-_._- 1.00

Baroness Schroeder --_-_-_-_-_-_-_- 2.50

Francis Willard _-_-_-_-_-_-_-_-- 7.50

Marie Lemoine -_-_-_-_-_-_-_-_-_-- 1.00

Early Light Pink:

Venus

Therese -

Midseason Light Pink:

Albert Crousse

Late Light Pink:

Grandiflora

Early Dark Pink:

Edulis Superba _-_-_-_-_-_-_-_-_-_-_-_ 1.00

M. J. Elie___- 2.50

Midseason Dark Pink:

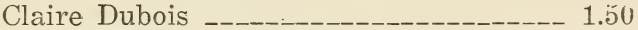

Walter Faxon -_-_-_-_-_-_-_-_-_.

Late Dark Pink:

Sarah Bernhardt _-_-_-_-_-_-_ 3.00

Humei _-_-_-_-_-_-_-_-_-_-_-_-_ .75

Early Red:

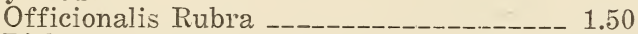

Richard Carvel

Midseason Red:

Felix Crousse --_-_-_-_-_-_-_-_-_-_-_ 1.50

Karl Rosenfield -

Mary Brand _-_-_-

Longfellow - -

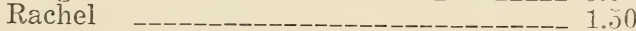


Kubra Nuperba -_-_-_-_-_-_-__-_-_. 1.0 ?

Meissonier _-_-_-_._-_._-_

Louis Van Houtte__-__-_. 75

Chas. McKellip _-_- 5.00

Late Red:

Dalachea

Unnamed Peonies, Red, White and Pink $50 \zeta$ ea.;

$\$ 1.40$ for three or $\$ 5 . C 0$ per clozen.

We are aiways glad to make suitable assortments for you of fine named varieties. We can furnish extra nice sorts at from $\$ 15.00$ to $\$ 25.00$ per dozen. Come and see our Peony fields during; June.

\section{Iris.}

We have a fine assortment. The more plentiful sorts run at $25 c$ each or $\$ 2.50$ per doz: $n$. while the finer kinds run from $\$ 5.00$ to $\$ 10$.C per dozen. We will always give you your money's worth in carefully named kinds.

\section{Phlox.}

One of the very finest perennials as they bloom all summer. Price $40 \%$ each or $\$ 4.50 \mathrm{per}$ dozen for field grown plants. Our assortment of fine named Phlox is very complete. R. P. Struth Cr's, ine cherry red and Ringstrom the large soit pink are leaders for landscape work.

\section{Evergreens.}

Balled and Burlapped Specimens.

12-18" 18-24" 2-21/2, 21/2-3, 3-4, $4-5$ "

Black Hills

Spruce___ $\$ 2.50 \$ 3.00 \$ 4.50 \$ 6.00 \$ 9.00 \$ 12.00$

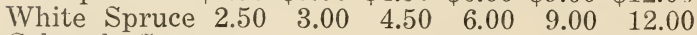
Colorado Spruce

(green) _-_ $2.50 \quad 3.00 \quad 4.50 \quad 6.00 \quad 9.00$

B.ue Colorado

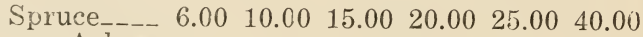

Chinese Artor

$\begin{array}{lllllll}\text { Vitae_.__- } 2.00 & 2.50 & 4.00 & 5.00 & 7.00 & 10.00\end{array}$

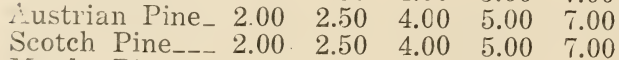

Mugho Pine

(Dwarf) _- $5.00 \quad 8.00$

Red Cedlar-_-

$\begin{array}{llll}4.00 & 5.00 & 7.00 & 10.60\end{array}$ 
Evergreens for Windbreak.

Red Cedars are best, and are always dependable.

\section{Each}

2 to 4 inch $--\$ .10$

6 to 12 inch

10 to 15 inch_- .40

12 to 18 inch -.75

18 to 24 inch_- 1.00

$\begin{array}{cccc}10 & \mathbf{1 0 0} & \mathbf{5 0 0} & \mathbf{1 0 0 0} \\ \$ .90 & \$ 8.00 & \$ 37.50 & \$ 70.00 \\ 2.75 & 25.00 & 115.00 & 225.00 \\ 3.50 & 30.00 & 140.00 & \\ 7.00 & 65.00 & 300.00 & \\ 9.00 & 85.00 & & \end{array}$

\section{Summer Blooming Bulbs.}
Each
Doz.
Cannas, leading varieties
$\$ .35$
$\$ 3.00$
Dahlia, leading varieties of the
Cactus, Decorative and Show_-_ .35
3.00
Gladiolus, named fine varieties_-_- $\quad .15 \quad 1.50$
Tuberoses

$.25 \quad 2.50$

Dutch Bulbs for Fall Planting.

Per 100 Doz.

Single and Double Early Tulips_-_-_\$8.00 \$1.5n

Darwin, Named _-_-_-_-_-_-_-_-_10.00 2.00

Darwin Mixed _-_-_-__-__-_-_-_ $6.00 \quad 1.00$

Crocus -

Hyacinths _-_-_-_-_-_-_-_-_25.00 3.00

We wish to call your special attention to our $\mathrm{XX}$ Special grade of fruit trees. They are the cream of the nursery and are sure to please the most exacting trade.

More and more each year the nursery business is being conducted on a cash basis and to encourage this, we will allow a credit of $10 \%$ on any of our fine Peonies if cash is paid at the time order is given. 


\section{INDEX.}

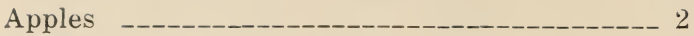

Apricots --_--_--_-_-_-_-_-_-_-_-_-_-_-_-_-_-_- 2

Asparagus _-_-_-_-_-_-_-_-_-_-_-_-_-_-- 3

Blackberries -_-_-_-_-_-_-_-_-_-_- 3

Bulbs ---_-_-_-_-_-_-_-_-_-_-_-_-_-_-_-_10

Cherries -_-_-_-_-_-_-_-_-_-_-_-_-_-_-_-_-_-- 1

Compass Cherry-Plum --_-_-_-_-_-_-_-_-_-_-- i

Crab Apples --_-_-_-_-_-_-_-_-_-_-_-_-_-_-_- 1

Currants _-_-_-_-_-_-_-_-_-_-_-_-_-_-_ 2

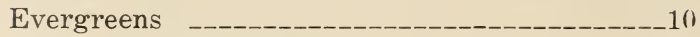

Forest Tree Seedlings__-_-__-_-__-_-_-_-_-_-_ 4

Gooseberries -_-_-_-_-_-_-_-_-_-_-_-_-_ 3

Grape Vines _-_-_-_-_-_-_ 2

Hansen Hybrid Plums__-__-_-_-_-_-_-_-_-_-_-- 1

Hedging --_-_-_-_-_-_-_-_-_-_-_-_-_-_-_--_ 4

Mulberries -_-_-_-_-_-_-_-_-_-_-_-_-_-_- 2

Niscellaneous -_-_-___-_-_-_-_-_-_-_-_-- 3

Peaches -_-_-_-_-_-_-_-_ 2

Pears -_-_-_-_-_-_-_-_-_-_-_-_-_-_-_-_-_-_ 2

Plums --_-_-_-_-_-_-_-_-_-_-_-_-_-_-_-_-_ 1

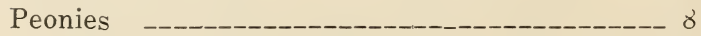

Perennials --_-_-_-_-_-_-_-_-__-_-_-_-_-_--- 7

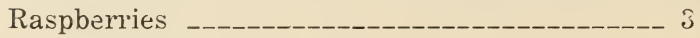

Rhubard -_-_-_-_-_-_-_-_-_-_-_-_-_-_ 3

Roses --_-_-_-_-_-_-_-_-_-_-_-_-_-_-_-_-_-_ 6

Shrubbery --_-_-_-_-_-_-_-_-_-_-_-_- 5

Shade and Ornamental Trees_-__-_-_-_-_-_-_-_- 3

Strawberries -_-_-_-_-_-_-_-_-_-_-_-_-_-_-- 3

Weepling Trees --_-_-_-_-_-_-_-_-_-_-_-_-_- 4

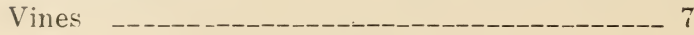





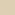

